\title{
Review Paper: Presurgical Language Mapping in Patients With Intractable Epilepsy: A Review Study
}

\author{
Mahdieh Karami ${ }^{1}$ (D), Jafar Mehvari Habibabadi ${ }^{\star}$ (D), Reza Nilipour ${ }^{3}$ (D), Majid Barekatain ${ }^{4}$ (D) William D. Gaillard ${ }^{5}$ (D), Hamid \\ Soltanian-Zadeh ${ }^{6,7}$ (iD) \\ 1. Institute for Cognitive Science Studies (ICSS), Tehran, Iran. \\ 2. Isfahan Neuroscience Research Center, Isfahan University of Medical Sciences, Isfahan, Iran. \\ 3. Department of Speech Therapy, University of Social Welfare and Rehabilitation Sciences, Tehran, Iran. \\ 4. Department of Psychiatry, School of Medicine, Isfahan University of Medical Sciences, Isfahan, Iran. \\ 5. Center for Neuroscience and Behavioral Health, Children's National Medical Center, George Washington University, Washington, D.C. USA. \\ 6. Departments of Communication, School of Electrical and Computer Engineering, University of Tehran, Tehran, Iran. \\ 7. Departments of Radiology and Research Administration, Henry Ford Health System, Detroit, MI, USA.
}

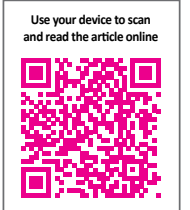

Citation: Karami, M., Mehvari Habibabadi, J., Nilipour, R., Barekatain, M., Gaillard, W. D., \& Soltanian-Zadeh, H. (2021) Presurgical Language Mapping in Patients With Intractable Epilepsy: A Review Study. Basic and Clinical Neuroscience, 12(2), 163-176. http://dx.doi.org/10.32598/bcn.12.2.2053.1

http://dx.doi.org/10.32598/bcn.12.2.2053.1

\section{Article info:}

Received: 20 Sep 2020

First Revision: 10 Oct 2020

Accepted: 08 Nov 2020

Available Online: 01 Mar 2021

Keywords:

Epilepsy, Brain mapping, Language, Functional Magnetic Resonance Imaging (fMRI), Persian

\begin{abstract}
A B S T RA C T
Introduction: about $20 \%$ to $30 \%$ of patients with epilepsy are diagnosed with drug-resistant epilepsy and one third of these are candidates for epilepsy surgery. Surgical resection of the epileptogenic tissue is a well-established method for treating patients with intractable focal epilepsy. Determining language laterality and locality is an important part of a comprehensive epilepsy program before surgery. Functional Magnetic Resonance Imaging (fMRI) has been increasingly employed as a non-invasive alternative method for the Wada test and cortical stimulation. Sensitive and accurate language tasks are essential for any reliable fMRI mapping.

Methods: The present study reviews the methods of presurgical fMRI language mapping and their dedicated fMRI tasks, specifically for patients with epilepsy.

Results: Different language tasks including verbal fluency are used in fMRI to determine language laterality and locality in different languages such as Persian. there are some considerations including the language materials and technical protocols for task design that all presurgical teams should take into consideration.

Conclusion: Accurate presurgical language mapping is very important to preserve patients language after surgery. This review was the first part of a project for designing standard tasks in Persian to help precise presurgical evaluation and in Iranian PWFIE.
\end{abstract}

\footnotetext{
* Corresponding Author:

Jafar Mehvari Habibabadi, PhD.

Address: Isfahan Neuroscience Research Center, Isfahan University of Medical Sciences, Isfahan, Iran.

Tel: +98 (912) 1302786

E-mail:drmehvari@hotmail.com
} 


\section{Highlights}

- Invasive and non invasive methods for presurgical language mapping in intractable epilepsy.

- Functional Magnetic Resonance Imaging (fMRI).

- Designing language tasks for mapping.

- Persian language tasks.

\section{Plain Language Summary}

People with intractable epilepsy can be treated by brain surgery. Their language and other cognitive abilities must be reserved after surgery, therefore, precise mapping is needed and it requires well-designed and standard tasks. Nowadays, functional magnetic resonance imaging is used as an invasive method for brain mapping. we reviewed presurgical language mapping tasks used in fMRI for patients with epilepsy.

\section{Introduction}

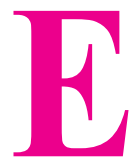

pilepsy is a group of disorders characterized by recurrent and unprovoked seizures that have been estimated as the most common neurological disorder found throughout the lifespan. Its prevalence is estimated to be about $1 \%$ worldwide (Fiest et al., 2017). A meta-analysis of 9 eligible studies from 2002 to 2010 concluded that the prevalence of life-long epilepsy and epilepsy-like syndromes in Iran was around 5\% (Sayehmiri, Tavan, Sayehmiri, Mohammadi, \& Carson, , 2014). However, a recent survey of epilepsy on 68038 Iranian subjects estimated the prevalence of epilepsy around 2\% (Pakdaman, Amini Harandi, Ashrafi, \& Alaeddini, 2018).

There are two broad categories of epilepsy based on the etiology and clinical manifestations: generalized and focal (Scheffer et al., 2017). Generalized epileptic seizures are conceptualized as rapidly engaging, bilaterally distributed networks and deep brain structures (Berg et al., 2010). Generalized epilepsies usually have genetic causes such as inherited mutations of neurotransmitter receptors or ion channel genes (Helbiga \& Tayounb, 2016).

On the other hand, a focal seizure originates in a neocortical region with a local spread that affects just one part of the brain; it may spread to one hemisphere or become secondarily generalized to the whole brain (Berg et al., 2010). The common causes of focal epilepsy are tumors, strokes, vascular malformations, post-traumatic gliosis, malformations of cortical development, mesial temporal sclerosis, brain infections, or surgical scars. Symptoms of focal seizures vary based on where the sei- zure originates and spreads (Skidmore, 2016). The new epilepsy classification (Figure 1) has been proposed by International League Against Epilepsy (ILAE) and added two more categories of "combined generalized and focal epilepsy” and “an unknown” (Scheffer et al., 2017)

When the seizure onset zone is detected in focal epilepsy, surgical resection of the epileptogenic tissue is a well-established method for treating Patients With Intractable Focal Epilepsy (PWIFE). For example, patients with refractory Temporal Lobe Epilepsy (TLE), the most common type of focal epilepsy in adults, are usually good candidates for epilepsy surgery (Wiebe, 2000; Zhang et al., 2015). The outcome of resection surgery in patients with a focus far from articulate areas can be excellent, especially in TLE cases. Epilepsy surgery for TLE has short and long-term seizure freedom of $85 \%$ and $50 \%-60 \%$, respectively (Wiebe, Blume, Girvin, Eliasziw, 2001).

The different surgical approaches and techniques for TLE surgery comprise standard anterior temporal lobectomy, electrocorticography-tailored temporal lobectomy, anteromedial temporal lobectomy, transcortical selective amygdalohippocampectomy, transsylvian selective amygdalohippocampectomy, sub-temporal selective amygdalohippocampectomy, temporal lobe disconnection, and hippocampal transection. They may have up to an $85 \%$ success rate (Al-Otaibi, Baeesa, Parrent, Girvin, \& Steven, 2012). The type of surgical approach for each patient is based on achieving the least complications while providing the most seizure control. The most important complications of epilepsy surgery are impairment of cognitive functions such as memory, vision, motor, and language. Therefore, presurgical evaluation of 


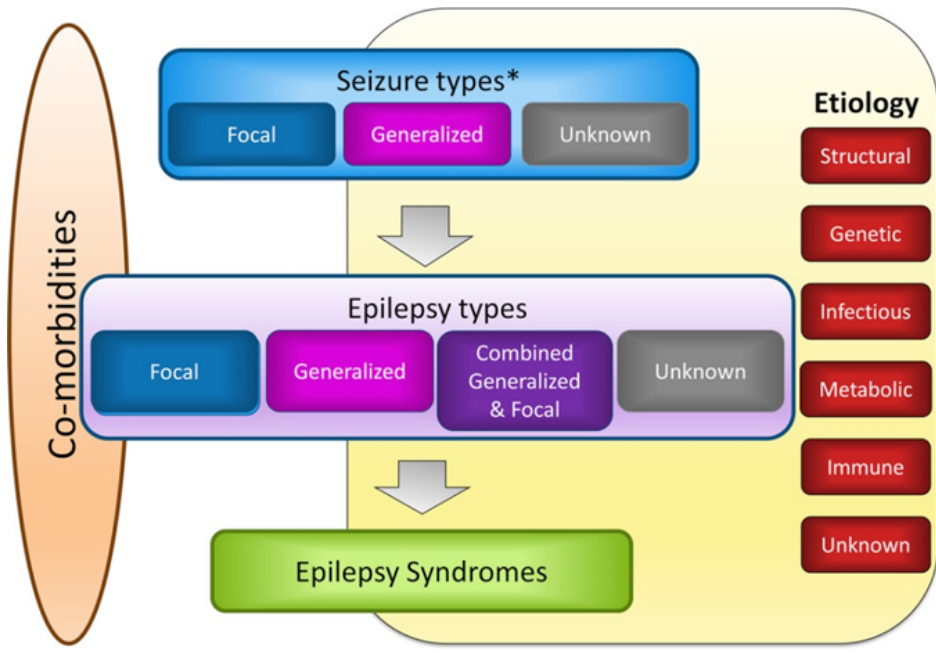

Figure 1. International League Against Epilepsy (ILAE) epilepsy classification

cognitive state has been one of the cornerstones of any comprehensive epilepsy program. Mehvari Habibabadi, Saba, Barekatain, Tabrizi, and Manoochehri (2018) conducted a prospective longitudinal study in Kashani Comprehensive Epilepsy Center, Isfahan City, Iran, from 2007 to 2017. They studied 214 patients with a diagnosis of intractable focal epilepsy for the postoperative outcome. They found high rates of seizure freedom after surgery in focal epilepsy patients $-81.8 \%$ of patients became seizure-free postoperatively.

\section{Presurgical Cognitive Assessment in Pa- tients With Epilepsy}

\subsection{Neuropsychological assessments}

Epilepsy has behavioral and cognitive consequences and comorbidities, which may decrease the quality of life. The cognitive manifestations of epilepsies may be polymorphic, pleomorphic, and diverse, whose basis has not been fully understood (Loughman, Seneviratne, Bowden, \& D'Souza, 2016). Up to $80 \%$ of patients with intractable focal epilepsy exhibit some cognitive deficits in presurgical neuropsychological testing. On the other hand, epilepsy surgery can be complicated by cognitive impairments revealed in postoperation neuropsychological evaluations. For example, anterior temporal lobectomy may result in a $45 \%$ decline in memory (Sherman et al., 2011; Helmstaedter, 2013). The main predictor of cognitive decline is presurgical neuropsychological performance, reflecting functional integrity of the pathological tissue in the epileptogenic zone and cognitive capacity of normal structures in the rest of the brain.

In a comprehensive epilepsy program, all patients with intractable focal epilepsy are evaluated for general intellec- tual function. These functions include attention, memory, executive functions, speed of information processing, language, visual-spatial capacity, sensory and motor functions, academic skills, personality, mood, form, and process of thought and behavior. In addition to the clinical interview, these domains should be assessed by a well-trained neuropsychologist with standardized and normative-based neuropsychological tests with vigorous psychometric properties (Wilson et al., 2015; Tavakoli et al., 2011).

The most widely-used tests in English for the evaluation of language in a presurgical setting are Boston Naming Test (BNT) and Western Aphasia Battery (WAB). Kaplan, Goodglass, and Weintraub introduced the BNT in 1983. It is a neuropsychological assessment tool to measure confrontational word retrieval in individuals with language disturbance. There are also several standard clinical linguistic tests developed in Persian to assess language impairments in patients with neurological diseases. The first standard Persian clinical linguistic test was based on the Bilingual Aphasia Test (BAT) format (Paradis, Paribakhat, \& Nilipour, 1987; Nilipour \& Ashayeri, 1989). It was initially developed for Persian and then for Azari and Kurdish (as the two major local languages spoken in Iran). The second test was the Persian Aphasia Battery (PAB) which was based on the format of BDAE (1972) and the scoring system of the BAT (Nilipour, 2010).

WAB was also designed to provide a means of evaluating the severity of language and cognitive impairments in neurological diseases (Shewan \& Kertesz, 1980). It is a highly valid and reliable clinical linguistic battery and provides Aphasia Quotient (AQ) as an index for the severity of language and cognitive impairments in neurological 
diseases. WAB has been developed and standardized for Persian (Nilipour, Pourshahbaz, \& Ghoreyshi, 2014).

The Aphasia Picture Naming Battery (Nilipour, 2002) and the P-WAB Battery (Persian bedside version AQ) (Nilipour et al., 2014) have been widely used for clinical linguistic evaluation of Persian-speaking CVA and PWIE patients in presurgical settings (Mehvari Habibabadi et al., 2018).

\subsection{Presurgical language mapping}

The Wada test, also known as the intracarotid sodium amobarbital procedure, was introduced to represent cerebral language and memory of each hemisphere in the late 1940s (Wada, 1960). During wada test, they inject a short acting anesthetic into the Internal Carotid Artery (ICA). This leads to temporarily anesthetizing one rain hemisphere, and then they test the other hemisphere for language and memory. Although wada test is considered as the gold standard to determine language laterality in PWIFE since 1960s (Loring \& Meador, 2015), it is an invasive method with considerable cost and rare but serious complications (stroke, bleeding). The Wada test has been gradually replaced by non-invasive techniques, especially functional Magnetic Resonance Imaging (fMRI), for language lateralization and mapping in presurgical settings (Loddenkemper, 2008; Gaillard et al., 2004). Language mapping by fMRI may establish language lateralization with a similar degree of success as the Wada testing (Binder, 2011; Szaflarski et al., 2017). fMRI is based on the observation that increased neuronal activity is associated with tightly regulated and regionally specific increases in cerebral blood flow (Fox \& Raichle, 1986). A change in magnetic resonance signal in the "activated" region occurs as a result of differences in the magnetic properties of oxygenated and deoxygenated hemoglobin, blood flow, and blood volume (Blood Oxygen Level-Dependent [BOLD] technique). Therefore, hemoglobin serves as an endogenous contrast agent for MRI. The mapping of neural networks involved in the performance of these tasks is possible by detecting the location of BOLD MR signal changes, which are induced during cognitive tasks (e.g. involving language, memory, and motor control) (Gaillard et al., 2004).

The difference in MR signals between a minimum of two conditions of experimental and control is considered as the base of experimental studies (Gaillard, 2004). EEG-fMRI combines the high temporal resolution of EEG signal with the high spatial resolution of blood BOLD MRI (Kay \& Szaflarski, 2014). In an EEG-fMRI study, Ebrahimzadeh, Soltanian-Zadeh, Araabi, Fesharaki, and Habibabadi (2019) extract the time series of epileptic components identified on EEG and fit them with a Generalized Linear Model (GLM). They revealed that this method allows precise and reliable localization of epileptic foci. Fernández et al. (2003) evaluated within-test and test-retest reliability of language fMRI in presurgical mapping in epileptic patients. They concluded that language fMRI was sufficiently reliable for determining global and regional lateralization of language representation.

In the presurgical settings, Benjamin et al. (2017) mentioned three distinct but related goals that are expected from language mapping using fMRI: language lateralization, language localization, and prediction of postsurgical language change.

\subsection{Language mapping in patients with Epilepsy}

To map language brain regions in patients with epilepsy, it is necessary to not only appreciate the neural correlates of language but also develop some clinically sensitive tasks for presurgical language mapping. Based on th Szaflarski e extended classical model of brain and language, articulated as the Wernicke-Lichtheim-Geschwind Model (1965), Broca area (pars triangularis and pars opercularis in the inferior frontal gyrus) is dedicated for language production and Wernicke area (posterior section of the superior temporal gyrus) for language comprehension. Their interconnecting white matter fibers (arcuate fasciculus, superior longitudinal fasciculus III, extreme capsule, uncinate) are dedicated to the interaction between the two mentioned regions. But later studies have revealed that language organization in the brain is more complex than this narrow localization model and involves cortical areas beyond the classical model. Broca and Wernicke areas are not the only brain involved areas in specific language skills, but they share similar functions. Broca area is involved in some forms of comprehension, and Wernicke in production. The new neuroscience evidence challenges not only the reliability of narrow localization models (Nilipour, 2018) but also proposes new models based on the neural network theories (Poeppel \& Hickok, 2004; Rauschecker \& Scott, 2009). For example, Stowea, Haverkortb, and Zwarts (2005) discussed four standard claims of classical models of language: 1 . There are only two primary language areas: Broca's and Wernicke's; 2. They are located in the left hemisphere; 3 . They are dedicated to distinct aspects of language processing; 4 . They are specific to language. They cast doubts on these claims based on new findings in neuroscience. fMRI studies by Benjamin et al. (2017) have identified six core clinically relevant language areas (that participate in aspects of language processing, but not all are critical to it): Broca area, Wernicke area (inferior, superior), Exner area, supplementary speech area, angular gyrus, and basal temporal language area (Figure 2). 


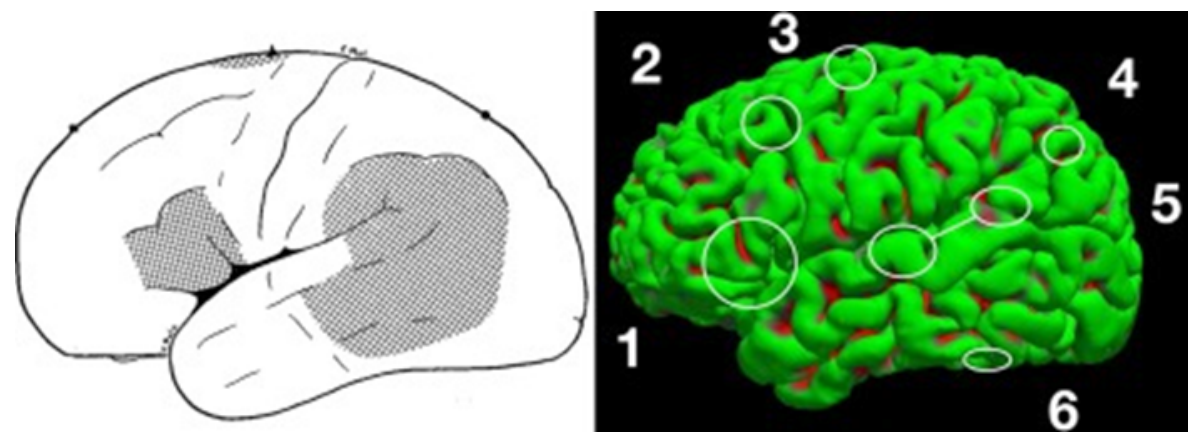

NEUR:SCIENCE

Figure 2. Six-core clinically relevant language areas

1: Broca area, in the posterior third of the inferior frontal gyrus; 2: Exner area, in the posterior middle frontal gyrus; 3: Supplementary motor area; 4: Angular gyrus; 5 : Wernicke area, inferior (mid to anterior STG), and superior (posterior STG and supramarginal gyrus) components; 6: Basal temporal language areas (Benjamine et al., 2017).

Also, considerable evidence from cortical stimulation and fMRI studies (Hamberger, 2007; Khoshkhouy Delshad, Nilipour, Barekatain, \& Oghabian, 2017) supports the possible role of hippocampus in naming functions. Although "Naming" is less anatomically specific, some studies revealed that confrontation naming ability depends on the integrity of the hippocampus and the connected frontotemporal networks (Bonelli et al., 2012). The perisylvian cortex mediates the naming function in the language dominant hemisphere (Bonelli et al., 2012).

As can be seen in Table 1, contemporary models predict different patterns of lateralization for different language processes (Hickok \& Poeppel, 2007; Peelle, 2012; Poeppel, 2014; Price, 2012). Although different models make various distinctions between language processes, some general patterns emerge. Acoustic processing of speech input and speech articulation is generally considered bilateral, whereas comprehension and generation of more meaningful language are considered to be lateralized (Bradshaw, Thompson, Wilson, Bishop, \& Woodhead, 2017). Epilepsy may lead to the displacement of language processing from the classical language areas. This phenomenon may result from a response to corti- cal insult, abnormal electrical circuitry, or underlying anatomic and functional anomalies in epilepsy (Chou, Serafini, \& Muh, 2018). Gaillard et al. (2011) has also stated that age of the onset and different etiologies of epilepsy are associated with varying frequencies of atypical language representation in the brain. For example, if damage occurs in the left middle cerebral artery territory at birth, it may lead to focal epilepsy, but there are no language deficits because of developmental neuroplasticity and compensation. In this case, language processing is being sustained entirely in the right hemisphere homologues. Thus, the earlier language representation is shifted, the more preserved function will be (there will not be any language impairment at the onset and later). On the other hand, if the damage happens slightly later, when language representation cannot shift, language impairments may appear. Atypical language is likely comorbid with epilepsy and may derive from etiology rather than as a consequence of epilepsy (Gaillard et al., 2004).

Drane and Pedersen (2019) mentioned different studies that show patients with seizure onset in the languagedominant cerebral hemisphere are more likely to experience baseline problems with naming (Drane et al.,

Table 1. Laterality in new language models (Bradshaw et al., 2017)

\begin{tabular}{|c|c|c|c|c|c|}
\hline Theoretical Principle/Model & $\begin{array}{l}\text { Speech Acoustic } \\
\text { Processing }\end{array}$ & $\begin{array}{c}\text { Speech } \\
\text { Comprehension }\end{array}$ & $\begin{array}{c}\text { Speech } \\
\text { Articulation }\end{array}$ & Semantics & Syntax \\
\hline $\begin{array}{l}\text { Dual-stream model of speech processing } \\
\text { (Hickok \& Poeppel, 2007) }\end{array}$ & B & B & L & B & - \\
\hline $\begin{array}{l}\text { Hierarchical asymmetry of linguistic complexity } \\
\text { (Peelle, 2012) }\end{array}$ & B & L & - & L & L \\
\hline $\begin{array}{l}\text { Bilateral sensorimotor inputs/outputs and left- } \\
\text { lateralized central language processes (Price, 2012) }\end{array}$ & B & $\mathrm{L}$ & B & $\mathrm{L}$ & $\mathrm{L}$ \\
\hline COM-PRE hypothesis (Poeppel, 2014) & B & L & B & L & L \\
\hline
\end{tabular}




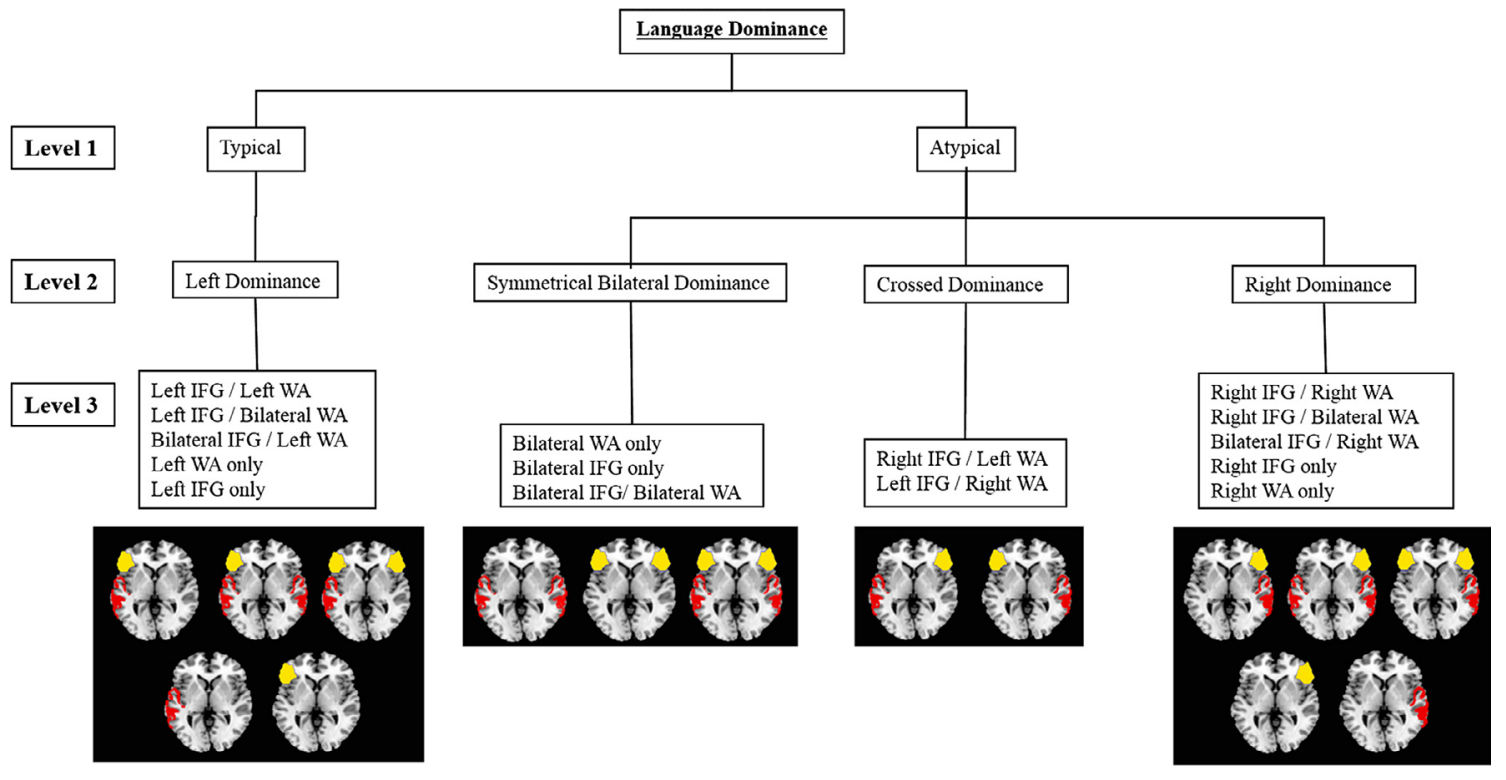

Figure 3. Laterality pattern (Berl et al., 2014)

NEUR SCIENCE

2015; Hermann, Davies, Foley, \& Bell, 1999; Langfit \& Rausch, 1996). Baseline deficits in verbal generative fluency tasks have also been reported in language-dominant TLE patients (Hermann et al., 1999; Martin, Loring, Meador, \& Lee, 1990).

While the language is supported by a typically left-lateralized, frontotemporal functional network in typically developing people (Bookheimer, 2002; Berl et al., 2014; Springer et al., 1999; Szaflarski et al., 2002), laterality patterns are different in epilepsy. Traditionally, three general and overarching patterns have been reported for atypical language organization in epilepsy patients (Balter, Lin, Leyden, Paul, $\&$ McDonald, 2019). The first pattern is a right hemisphere dominance, in which core language expression and comprehension networks are typically located in the left hemisphere and most often shifting to the homologous regions in the right hemisphere. This pattern is also seen in 3\%-4\% of typically developing controls (Gaillard et al., 2002, 2004, 2007; Thivard et al., 2005). The second pattern is crossed or bilateral dominance. In this pattern, while some language functions move to the right hemisphere, some language representations are preserved in the left hemisphere (Baciu et al., 2001; Thivard et al., 2005). The intrahemispheric reorganization is also considered as the third pattern of atypical language reorganization in epilepsy. Language regions shift (but not very far) within the same hemisphere that contains the seizure onset zone. Language regions often shift to regions adjacent to the typical perisylvian cortex in the frontal or temporal lobe (Bell et al., 2002; Brazdil, Zakopcan, Kuba, Fanfrdlova, \& Rektor, 2003; Mbwana et al., 2009). Generally, atypical language is reported in $4 \%$ of healthy subjects and $30 \%$ of patients with epilepsy.
However, Berl et al. (2014) believed that this classification was oversimplified. They conducted a data-driven clustering method on 220 epilepsy patients performing an auditory semantic decision task. They hypothesized 15 possible patterns of language representation (Figure 3). They indicated that right lateralization is more common in patients with a left-sided seizure focus. In this study, patients have more bilateral Inferior Frontal Gyrus (IFG) activation than bilateral Wernicke Area (WA) activation. Patients with left-handed seizures were more likely to show right-lateralized IFG, while patients with an early age of seizure onset were more likely to show bilateral WA, and patients with a left-sided seizure focus were more likely to show a unilateral right (IFG and WA) pattern of language dominance.

Balter et al. (2019) considered Berl et al.'s (2014) study of note that while much of the literature in fMRI and epilepsy continues to treat language as a monolithic construct in which all aspects of language either shift to the right hemisphere or remain left-lateralized. Berl et al. (2014) overcame this limitation by presenting language as a multivariate construct made up of different components and discrete functionalities that might reorganize together or independently.

\subsection{Task design for language mapping using fMRI}

Proper and sensitive task design in language mapping is an essential step. Most fMRI language studies employ a block design of three or more cycles of control and experimental conditions, each lasting between 20 and 
40 seconds (Gaillard et al., 2004). Language tasks are presented either in auditory or visual (written) form. The stimuli of each task and control condition must have sufficient distinction to elicit a hemodynamic response (Gaillard et al., 2004) and laterality measure.

Bradshaw et al. (2017) assessed evidence on the robustness of laterality measured using fMRI with different language tasks for healthy monolingual adults from studies published between 2000 and 2016. They reported that verbal fluency tasks had been considered the most commonly used and the easiest standard for measuring language laterality with fMRI (Bradshaw et al., 2017). Verbal fluency paradigms identify dorsolateral prefrontal networks involved in language processing (Petersen, Fox, Posner, Mintun, Raichle, 1988; Pujol, Deus, Losilla, \& Capdevila, 1999; Gaillard, 2004). A research showed that the verbal fluency paradigm is selected for its ease of understanding for a wide range of patients with epilepsy with different levels of cognitive abilities. It has been shown to lateralize expressive language reliably (Bonelli et al., 2012). However, the verbal fluency task is autoparametric and cannot be used for children under seven. There are different forms of verbal fluency tasks in which the participant must generate (covertly or overtly) words that meet specific criteria, including words beginning with a particular letter (phonemic fluency), words belonging to a particular semantic category (semantic fluency), verbs that are semantically associated with a particular noun (verb generation, which is reported to be more reliable and gives more temporal activation), or words that are antonyms/synonyms (antonym/synonym generation) (Bradshaw et al., 2017).

Tasks that require integration across the semantic content of different concepts (e.g. semantic relatedness decision), rather than simple category membership decision on single words, lead to stronger laterality, and naming from description yields more robust laterality measurement than naming from pictures. In other words, adding sentence comprehension (oral or written) component seems to improve the lateralizing power of the task (Bradshaw et al., 2017). It not just improves lateralizing but also gets frontal and temporal regions identified by (Gaillard et al., 2004) that examined developmental differences, in location and extent of fMRI language activation maps, between adults and children while performing a semantic fluency task. The results showed that brain areas that process semantic verbal fluency are similar in children and adults. It is reported that verbal fluency tasks tend to induce the strongest laterality in frontal ROIs (Gaillard et al., 2004) and also strong laterality in temporoparietal ROIs when the activation is present (Bradshaw et al., 2017). The activation in temporal lobes is weaker for verbal fluency tasks, less so with the noun-verb generation, but the sentence tasks give better temporal activation.

The most frequently used TLE language task (usually for cortical stimulation and postoperative assessment) is object naming (Drane \& Pedersen, 2019). Studies usually examined visual confrontation naming ability, including human-made objects (Hermann et al., 1999; Ives-Deliperi $\&$ Butler, 2012), and some studies have explored a widerrange of object type or used naming to describe paradigm (Hamberger \& Tamny, 1999; Hamberger, Goodman, Perrine, \& Tamny, 2001). Although the naming is a complex linguistic function that requires both receptive and expressive components, with access to both semantics and phonology, it yields low LI values (Bradshaw et al., 2017), and little frontal activation is seen if you do something with the pictures, for example, animals. Evidence suggests that naming from description yields more robust laterality measurement than naming from pictures since additional sentence comprehension component appears to improve the lateralizing power of the task (Bradshaw et al., 2017). For instance, Gaillard et al. (2002) studied 30 patients with TLE, in which they silently named an object described by a sentence compared to a visual control. They concluded that this task could identify language dominance in frontal and temporal areas.

Comparison of word generation with sentence generation showed stronger laterality for processing connected sentences over single words (Peelle, 2012; Poeppel, 2014; Gaillard et al., 2004). Regarding semantic decision tasks, stronger laterality can be observed when tasks require integration across the semantic content of different concepts (e.g. semantic relatedness decision), rather than simple category membership decision on single words (Bradshaw et al., 2017).

Gaillard et al. (2004) emphasized using a panel of fMRI language paradigms. They stated that having a panel of tasks may help evaluate partial epilepsy patients than a single task. It also reduces the likelihood of nondiagnostic findings, improves interrater reliability, and redundancy helps confirm findings. Since some specific cognitive impairments are common in patients with epilepsy, it is essential to ensure that patients can perform the imaging tasks (Gaillard et al., 2004).

Studies on healthy subjects reveal mixed findings between cortical activation and task performance or task difficulty in language-related paradigms (Weber et al., 2006). Weber et al. (2006) observed differential effects of task per- 
formance on the volume of activation in the inferior frontal and the temporoparietal region of interest, but performance measures did not correlate with the lateralization of activation. Accordingly, they mentioned that laterality seems to be independent of performance - at least as long as individual thresholding is used. Others find that effort may be more important than performance (Gaillard et al., 2004).

LI can be based on activated voxels or magnitude of activation (or both). Most studies use activated voxels. The more difficult the tasks are, the more bilateral activation is seen. Task difficulty and input modality, visual or auditory, might also influence the hemispheric distribution of activated regions (Seghier, 2008). Carpentier et al. (2001) used two parallel cognitive language tasks for TLE patients and a control group. In one of the tasks, he used auditory input and in the other visual input. Language lateralization scores of the visual task were stronger than for the auditory task. Repetition tasks can also affect LI and lead to a pseudo increase in bilaterality (Lohmann, Deppe, Jansen, Schwindt, \& Knech, 2004).

Control condition or baseline selection is also crucial in interpreting the results (Stark \& Squire, 2001). The control condition should control for nonlinguistic aspects of a task, for example, attention, first- and secondorder auditory processing, or motor controls, if having to push a button in a monitored response. Peck et al. (2004) showed that activity in language areas (e.g. Broca's area) was dependent on the baseline used. More activation was seen in these areas when the baseline was passive viewing of nonsense objects than picture naming. Using a low-level baseline condition (e.g. rest or fixation condition) may lead to LI values near to zero (i.e. more bilateral functional patterns), while the elimination of non-relevant regions involved in early perceptual and sensory processing of perceptual baseline may lead to higher LI values (Seghier, 2008; Hund-Georgiadis, Lex, \& von Cramon, 2001). Ideally, control conditions should have bilateral activation patterns (i.e. $|\mathrm{LI}| \cong 0$ ) because if control activation has, for example, a strong RH dominance, the activation task might be artificially lateralized to the LH (LI towards +1 as all right activations have been removed by the control condition) (Seghier, 2008).

In verbal fluency tasks, an individual may show a stronger LI when an active baseline (active baselines such as finger tapping or silent word repetition versus passive baseline task such as fixation) is used (Dodoo-Schittko, Rosengarth, Doenitz, \& Greenlee, 2012). Bradshaw et al. (2017) asked how one should decide which of these can best reflect the "true" laterality. They stated that the greater strength of laterality with an active baseline is often taken to indicate that this is a more accurate laterality measurement. However, other metrics such as the reliability of the laterality and in clinical work its predictiveness of postsurgical outcomes may arguably represent better standards for assessing goodness of laterality measurement.

\subsection{Risks of postsurgical language impairment}

Language impairment is considered a critical risk of surgery; therefore, accurate mapping of the language cortex is of great importance. In patients with epilepsy, functional anatomy may be reorganized with a transfer of functions to other areas in the ipsilateral or contralateral hemisphere (Ni, Wang, Yu, Wu, \& Wang, 2019; Berl et al., 2005). About $25 \%-60 \%$ of adults who undergo Anterior Temporal Lobectomy (ATL) will suffer from some form of dysnomia because some temporal lobe structures targeted for resection play a significant role in the language (Sabsevitz et al., 2003). The phenomenon has also been reported in patients after non-dominant ATL (Jabbour, Hempel, Gates, Zhang, \& Risse, 2005). Davies et al. (1998) reported that approximately $30 \%$ of left TLE patients develop a significant decline in naming abilities after surgery. Schwarz and Pauli (2009) reported a significant association between postoperative naming decline and impaired semantic functions in epilepsy surgery.

Davies et al. (1998) also reported that up to $40 \%$ of patients would develop language deficits after ATL, especially a decline in naming ability. A decline in verbal fluency has also been reported in LTLE patients (Helmstaedter, Gleibner, Zentner, \& Elger, 1998). Frontal lobe epilepsy and its surgical treatment have also been associated with verbal generative fluency deficits (Drane \& Pedersen 2019; Risse, 2006; Sarkis et al., 2013). Generally speaking, language deficits of some forms (including naming) is seen after lobectomy, most often in dominant (for most left) temporal resection.

In a recent study, You et al. (2019) aimed to predict language deficits after epilepsy surgery. Thirty-five TLE adults completed a preoperative fMRI auditory description decision task. They also used BNT to determine language functioning before and after surgery. Their final regression model predicted $38 \%$ of the naming score change variance. They revealed that the top $10 \%$ of language fMRI activation provides additional predictive power and should be considered in presurgical planning. 


\subsection{Challenges of designing language mapping task in Persian}

Different studies in language mapping with fMRI revealed somehow similar activation maps. The activation maps are more similar than different, especially for auditory processing (Dodoo-Schittko et al., 2012). Minor idiosyncrasies of a given language do not change fundamental activation patterns. However, compatibility with cultural and linguistic features of the fMRI tasks in each language is one of the most critical issues that should be considered in task design selection and stimuli. Mere translation of existing tasks from one language into another may not necessarily result in accurate brain mapping. For example, the Chinese language differs from alphabetical languages as it is an ideographic script, requiring one to memorize the phonology and meaning of each character to vocalize and comprehend. Thus, brain activation in processing the written Chinese language may differ from that in processing English (Ni et al., 2019). Task design for presurgical mapping for Persianspeaking patients should be designed incorporating linguistic and cultural features such as proper task design and selection of language-specific stimuli based on their psycholinguistic features (Momenian, Nilipour, Ghafar Samara, Oghabian, \& Cappa, 2016).

Persian is a member of the Indo-European language family and a branch of the Indo-Iranian group of languages. With respect to language-specific features, Persian is an SOV language with a rather loose syntax because of its direct object /ra/ and indirect object marker /be/ (Samvelian, 2018). Modern Persian script is alphabetic but was originally borrowed from Arabic in the ninth century and has been modified by adding four new characters to represent four Persian phonemes (/p/, /ch/, $/ \mathrm{zh} /, / \mathrm{g} /$ ) not found in the Arabic phonological system (Karimi \& Nilipour, 2011).

Because of the major structural differences between Persian and Arabic, there is rarely a one-to-one correspondence between the graphemic and phonemic representation of words which causes ambiguity in pronouncing, understanding the meaning, and reading words. There is no graphemic representation for short vowels (e, a, o), but long vowels /i, u, aa/ do have a graphemic representation. Verbs carry a rather complex morphology to express tense, aspect, mood, number, and person but nouns, are only inflected for number.

As Nilipour (2018) has indicated, different languages have different conceptual, phonological, and morphosyntactic properties. Therefore, one would expect to see dif- ferent patholinguistic symptoms if the same lesion affects similar brain regions in bilingual or multilingual patients (Nilipour \& Ashayeri, 1989; Nilipour, \& Paradis, 1995). Based on the results of systematic neuroimaging studies on Persian-speaking subjects, the tasks and the stimuli implemented in language mapping should be controlled for language-specific features and psycholinguistic properties of the stimuli as in Momenian et al. (2016) study.

With respect to language mapping tasks in Persian, Nilipour (2018) reviewed four recently-published Persian neuroimaging studies. The main objective of the first two neuroimaging studies (Mahdavi et al. 2008, 2010) was to design protocols for presurgical clinical applications using word production, reverse word reading, and word generation as their tasks. The authors reported robust activation of classical language areas in all paradigms except in the object naming task. But so far, there are no published presurgical applications based on these tasks.

In the third study (Mahdavi et al., 2010), the objective was to differentiate cortical activation of Persian as compared with English in the healthy bilinguals, but they did not use the same tasks in both languages. They employed word production and reverse word reading for Persian and word generation for English. They indicated that common regions were activated by both Persian and English stimuli, specifically in the left inferior frontal gyrus and other perisylvian areas. They also reported activation in RH occipital cortex.

Nilipour argues that one inconsistency in task design in this study is that, despite using different tasks in Persian and English, they reported activation of the same common regions (Nilipour, 2018: p:456). There is also no evidence of normative data and considerations of psycholinguistic and language-specific features of the selected stimuli in designing language tasks adapted for these neuroimaging experiments for Persian.

Another recent fMRI study was reported by Nilipour (2018) is Momenian et al. (2016) on neural correlates of Persian object and action naming in 15 healthy native speakers of Persian. One novelty about task design in this study as compared to previous naming studies in other languages is the application of a cued-covert sentence completion to name each object and action to tease out inflectional features of verbs in context as a Persian specific feature.

The second novelty of this study in Persian was that the stimuli were selected from two different normed data sets. Objects were selected from Bakhtiar et al.,'s study a study based on psycholinguistic features of three differ- 
ent categories (living, non-living, and tools) and actions were selected from as a combination of common transitive and intransitive high-frequency verbs in Persian from a data set (Nilipour et al., 2018).

In addition to the studies mentioned above, two recent studies have been reported in Persian. Batouli, Hasani, Gheisari, Behzad, and Oghabian (2016) investigated the influence of several factors on language LI, using data from 120 Persian-speaking patients who underwent fMRI for presurgical planning. They used two language tasks, including word generation and reverse word reading. The results indicated that the left hemisphere was dominant for language function, although a higher LI was obtained using the word generation task.

Khoshkhouy Delshad et al. (2017) studied neural correlates of visual confrontation naming in Persian-speaking subjects. They found that activation in the left hippocampus was associated with visual confrontation naming performance. Participants with higher scores showed greater fMRI activation in their left hippocampus.

\section{Conclusion}

The general principles of fMRI brain mapping for language are appraised in this review. This knowledge provides a foundation for future recommendations to design standard sensitive tasks for language mapping in Persian-speaking PWIFE. These tasks will be employed for fMRI mapping in the presurgical and postsurgical assessment. Nevertheless, these tests might consider the essential features of the Persian language for a precise mapping of the Persian language. This paper is the first step of a clinical project for designing the standard presurgical language mapping tasks for the assessment and rehabilitation of Persian-speaking PWIFE.

\section{Ethical Considerations}

\section{Compliance with ethical guidelines}

This article is a meta-analysis with no human or animal sample.

\section{Funding}

This research did not receive any grant from funding agencies in the public, commercial, or non-profit sectors.

\section{Authors' contributions}

Conceptualization and supervision, review and editing the draft: All authors; Writing original draft: Mahdieh Karami.

\section{Conflict of interest}

The authors declared no conflict of interest.

\section{Acknowledgments}

The authors would like to thank the Cognitive Science and Technology Council of Iran, National Brain Mapping Laboratory of Iran, Isfahan Comprehensive Epilepsy Center; the Isfahan University of Medical Science, and Children's National Hospital, George Washington University, USA.

\section{References}

Al-Otaibi, F., Baeesa, S. S., Parrent, A. G., Girvin, J. P., \& Steven, D. (2012). Surgical techniques for the treatment of temporal lobe epilepsy. Epilepsy Research and Treatment, 2012, 374848. [DOI:10.1155/2012/374848] [PMID] [PMCID]

Baciu, M., Kahane, P., Minotti, L., Charnallet, A., David, D., \& Le Bas, J. F., et al. (2001). Functional MRI assessment of the hemispheric predominance for language in epileptic patients using a simple rhyme detection task. Epileptic Disorders, 3(3), 117-24. https://www.jle.com/en/revues/epd/e-docs/functional_mri_assessment_of_the_hemispheric_predominance_ for_language_in_epileptic_patients_using_a_simple_rhyme_ detection_task_110091/article.phtml?cle_doc=0001AE0B

Balter, S., Lin, G., Leyden, K. M., Paul, B. M., \& McDonald, C. R. (2019). Neuroimaging correlates of language network impairment and reorganization in temporal lobe epilepsy. Brain and Language, 193, 31-44. [DOI:10.1016/j.bandl.2016.06.002] [PMID] [PMCID]

Batouli, S. A. H., Hasani, N., Gheisari, S., Behzad, E., \& Oghabian, M. A. (2016). Evaluation of the factors influencing brain language laterality in presurgical planning. Physica Medica, 32(10), 1201-9. [DOI:10.1016/j.ejmp.2016.06.008] [PMID]

Bell, B., Hermann, B., Seidenberg, M., Davies, K., Cariski, D. Rosenbek, J., et al. (2002). Ipsilateral reorganization of language in early-onset left temporal lobe epilepsy. Epilepsy $\mathcal{E}$ Behavior, 3(2), 158-64. [DOI:10.1006/ebeh.2002.0322] [PMID]

Benjamin, C. F., Walshaw, P. D., Hale, K., Gaillard, W. D., Baxter, L. C., \& Berl, M. M., et al. (2017). Presurgical language fMRI: Mapping of Six critical regions. Human Brain Mapping, 38, 4239-55. [DOI:10.1002/hbm.23661] [PMID] [PMCID]

Berg, A. T., Berkovic, S. F., Brodie, M. J., Buchhalter, J., Cross, J. H. \& van Emde Boas, W., et al. (2010). Revised terminology and concepts for organization of seizures and epilepsies: report of the ILAE Commission on Classification and Terminology, 2005-2009. Epilepsia, 51(4), 676-85. [DOI:10.1111/j.1528-1167.2010.02522.x] [PMID] 
Berl, M. M., Balsamo, L. M., Xu, B., Moore, E. N., Weinstein, S. L., \& Conry, J. A., et al. (2005). Seizure focus affects regional language networks assessed by fMRI. Neurology, 65(10), 1604-11. [DOI:10.1212/01.wnl.0000184502.06647.28] [PMID]

Berl, M. M., Zimmaro, L. A., Khan, O. I., Dustin, I., Ritzl, E., \& Duke, E. S., et al. (2014). Characterization of atypical language activation patterns in focal epilepsy. Annals of Neurology, 75(1), 33-42. [DOI:10.1002/ana.24015] [PMID] [PMCID]

Binder, J. R., Gross, W. L., Allendorfer, J. B., Bonilha, L., Chapin, J., \& Edwards, J. C., et al. (2011). Mapping anterior temporal lobe language areas with fMRI: A multicenter normative study. Neuroimage, 154(2), 1465-75. [DOI:10.1016/j.neuroimage.2010.09.048] [PMID] [PMCID]

Bookheimer, S. (2002). Functional MRI of language: new approaches to understanding the cortical organization of semantic processing. Annual Review of Neuroscience, 25(1), 151-88. [DOI:10.1146/ annurev.neuro.25.112701.142946] [PMID]

Bonelli, S. B., Thompson, P. J., Yogarajah, M., Vollmar, C., Powell, R. H., \& Symms, M. R., et al. (2012). Imaging language networks before and after anterior temporal lobe resection: Results of a longitudinal fMRI study. Epilepsia, 53(4), 639-50. [DOI:10.1111/j.15281167.2012.03433.x] [PMID] [PMCID]

Brazdil, M., Zakopcan, J., Kuba, R., Fanfrdlova, Z., \& Rektor, I. (2003). Atypical hemispheric language dominance in left temporal lobe epilepsy as a result of the reorganization of language functions. Epilepsy E Behavior, 4(4), 414-9. [DOI:10.1016/S1525-5050(03)00119-7]

Bradshaw, A. R., Thompson, P. A., Wilson, A. C., Bishop, D. V., \& Woodhead, Z. V. (2017). Measuring language lateralisation with different language tasks: A systematic review. Peer I, 5, e3929. [DOI:10.7717/peerj.3929]

Carpentier, A., Pugh, K. R., Westerveld, M., Studholme, C., Skrinjar, O., \& Thompson, J. L., et al. (2001). Functional MRI of language processing: dependence on input modality and temporal lobe epilepsy. Epilepsia, 42(10), 1241-54. [DOI:10.1046/ j.1528-1157.2001.35500.x] [PMID]

Chou, N., Serafini, S., \& Muh, C. R. (2018). Cortical language areas and plasticity in pediatric patients with epilepsy: A review. Pediatric Neurology, 78, 3-12. https://www.sciencedirect.com/science/ article/pii/S0887899417301182

Davies, K. G., Bell, B. D., Bush, A. J., Hermann, B. P., Curtis Dohan Jr F., \& Jaap, A. S. (1998). Naming decline after left anterior temporal lobectomy correlates with pathological status of resected hippocampus. Epilepsia, 39(4), 407-19. [DOI:10.1111/j.1528-1157.1998. tb01393.x] [PMID]

Dodoo-Schittko, F., Rosengarth, K., Doenitz, C., \& Greenlee, M. W. (2012). Assessing language dominance with functional MRI: The role of control tasks and statistical analysis. Neuropsychologia 50(11), 2684-91. https:/ / www.sciencedirect.com/science/article/ abs/pii/S0028393212003284

Drane, D. L., \& Pedersen, N. P. (2019). Knowledge of language function and underlying neural networks gained from focal seizures and epilepsy surgery. Brain and Language, 189, 20-33. [DOI:10.1016/j.bandl.2018.12.007] [PMID] [PMCID]

Drane, D. L., Loring, D. W., Voets, N. L., Price, M., Ojemann, J. G., \& Willie, J. T., et al. (2015). Better object recognition and naming outcome with MRI-guided stereotactic laser amygdalohippocampotomy for temporal lobe epilepsy. Epilepsia, 56(1), 101-13. [DOI:10.1111/epi.12860] [PMID] [PMCID]
Ebrahimzadeh, E., Soltanian-Zadeh, H., Araabi, B. N., Fesharaki, S. S. H., \& Habibabadi, J. M. (2019). Component-related BOLD response to localize epileptic focus using simultaneous EEG-fMRI recordings at 3T. Journal of Neuroscience Methods, 322, 34-49. [DOI:10.1016/j.jneumeth.2019.04.010] [PMID]

Fernandez, G., Specht, K., Weis, S., Tendolkar, I., Reuber, M., \& Fell, J., et al. (2003). Intrasubject reproducibility of presurgical language lateralization and mapping using fMRI. Neurology, 60(6), 969-75. [DOI:10.1212/01.WNL.0000049934.34209.2E] [PMID]

Fiest, K. M., Sauro, K. M., Wiebe, S., Patten, S. B., Kwon, C. S., \& Dykeman, J., et al. (2017). Prevalence and incidence of epilepsy: A systematic review and meta-analysis of international studies. Neurology, 88(3), 296-303. [DOI:10.1212/ WNL.0000000000003509] [PMID] [PMCID]

Fox, P. T. \& Raichle, M. E. (1986). Focal physiological uncoupling of cerebral blood flow and oxidative metabolism during somatosensory stimulation in human subjects. Proceedings of the National Academy of Science, 83(4), 1140-44. [DOI:10.1073/ pnas.83.4.1140] [PMID] [PMCID]

Gaillard, W. D., Pugliese, M., Grandin, C. B., Braniecki, S. H., Kondapaneni, P., \& Hunter, K., et al. (2001). Cortical localization of reading in normal children: An fMRI language study. Neurology, 57(1), 47-54. [DOI:10.1212/WNL.57.1.47] [PMID]

Gaillard, W. D., Berl, M. M., Moore, E. N., Ritzl, E. K., Rosenberger, L. R., \& Weinstein, S. L., et al. (2007). Atypical language in lesional and nonlesional complex partial epilepsy. Neurology, 69(18), 1761-71. [DOI:10.1212/01.wnl.0000289650.48830.1a] [PMID]

Gaillard, W. D., Balsamo, L., Xu, B., McKinney, C., Papero, P. H., \& Weinstein, S., et al. (2004). fMRI language task panel improves determination of language dominance. Neurology 63(8), 1403-8. [DOI:10.1212/01.WNL.0000141852.65175.A7] [PMID]

Gaillard, W. D., Balsamo, L., Xu, B., Grandin, C. B., Braniecki, S. H., \& Papero, P. H., et al. (2002). Language dominance in partial epilepsy patients identified with an fMRI reading task. Neurology, 59(2), 256-65. [DOI:10.1212/WNL.59.2.256] [PMID]

Gaillard, W. D., Sachs, B. C., Whitnah, J. R., Ahmad, Z., Balsamo, L. M., \& Petrella, J. R., et al. (2003). Developmental aspects of language processing: fMRI of verbal fluency in children and adults. Human Brain Mapping, 18(3), 176-85. [DOI:10.1002/ hbm.10091] [PMID] [PMCID]

Gaillard, W. D. (2004). Functional MR imaging of language, memory, and sensorimotor cortex. Neuroimaging Clinics, 14(3), 471-85. [DOI:10.1016/j.nic.2004.04.005] [PMID]

Kaplan, E., Goodglass, H., Weintraub, S. (1983). Boston Naming Test. Philadelphia: Lea \& Febiger. [DOI:https://doi. org/10.1007/978-0-387-79948-3_869]

Geschwind, N. (1965). Disconnexion syndromes in animals and man. Brain, 88(3), 585. [DOI:10.1093/brain/88.3.585] [PMID]

Hamberger, M. J., \& Tamny, T. R. (1999). Auditory naming and temporal lobe epilepsy. Epilepsy Research, 35(3), 229-43. [DOI:10.1016/S0920-1211(99)00023-6]

Hamberger, M. J. (2007). Cortical language mapping in epilepsy: A critical review. Neuropsychology Review, 17(4), 477-89. https://link.springer.com/article/10.1007/s11065-007-9046-6 
Hamberger, M. J., Goodman, R. R., Perrine, K., \& Tamny, T. (2001). Anatomic dissociation of auditory and visual naming in the lateral temporal cortex. Neurology, 56(1), 56-61. [DOI:10.1212/WNL.56.1.56] [PMID]

Helmstaedter, C. (2013). Cognitive outcomes of different surgical approaches in temporal lobe epilepsy. Epileptic Disorders, 15(3), 221-39. https://link.springer.com/article/10.1684/ epd.2013.0587

Helmstaedter, C., Gleibner, U., Zentner, J., \& Elger, C. E. (1998). Neuropsychological consequences of epilepsy surgery in frontal lobe epilepsy. Neuropsychologia, 36(4), 333-41. [DOI:10.1016/S0028-3932(97)00118-8]

Hermann, B., Davies, K., Foley, K., \& Bell, B. (1999). Visual confrontation naming outcome after standard left anterior temporal lobectomy with sparing versus resection of the superior temporal gyrus: A randomized prospective clinical trial. Epilepsia, 40(8), 1070-6. [DOI:10.1111/j.1528-1157.1999.tb00821.x] [PMID]

Helbig, I., \& Abou Tayoun, A. N. (2016). Understanding genotypes and phenotypes in epileptic encephalopathies. Molecular Syndromology, 7(4), 172-81. [DOI:10.1159/000448530] [PMID] [PMCID]

Hickok, G., \& Poeppel, D. (2004). Dorsal and ventral streams: a framework for understanding aspects of the functional anatomy of language. Cognition, 92(1-2), 67-99. [DOI:10.1016/j. cognition.2003.10.011] [PMID]

Hickok, G., \& Poeppel, D. (2007). The cortical organization of speech processing. Nature Reviews Neuroscience, 8(5), 393-402. [DOI:10.1038/nrn2113] [PMID]

Hund-Georgiadis, M., Lex, U., \& von Cramon, D. Y. (2001). Language dominance assessment by means of fMRI: Contributions from task design, performance, and stimulus modality. Journal of Magnetic Resonance Imaging: An Official Journal of the International Society for Magnetic Resonance in Medicine, 13(5), 668-75. [DOI:10.1002/jmri.1094]

Ives-Deliperi, V. L., \& Butler, J. T. (2012). Naming outcomes of anterior temporal lobectomy in epilepsy patients: A systematic review of the literature. Epilepsy \& Behavior, 24(2), 194-198. [DOI:10.1016/j.yebeh.2012.04.115] [PMID]

Jabbour, R. A., Hempel, A., Gates, J. R., Zhang, W., \& Risse, G. L. (2005). Right hemisphere language mapping in patients with bilateral language. Epilepsy \& Behavior, 6(4), 587-92. [DOI:10.1016/j.yebeh.2005.03.008] [PMID]

Karimi, H., \& Nilipour, R. (2011). Chapter 8: Characteristics of Developmental Stuttering in Iran. In P. Howell, J. Van Borsel (Eds.), Multilingual Aspects of Fluency Disorders. Bristol, Blue Ridge Summit: Multilingual Matters. [DOI:10.21832/9781847693570-010] [PMCID]

Kay, B., \& Szaflarski, J. P. (2014). EEG/fMRI contributions to our understanding of genetic generalized epilepsies. Epilepsy $\mathcal{E}$ Behavior, 34, 129-35. [DOI:10.1016/j.yebeh.2014.02.030] [PMID] [PMCID]

Khoshkhouy Delshad, H., Nilipour, R., Barekatain, M., \& Oghabian, M. A. (2017). Neural correlates of visual confrontation naming in persian-speaking individuals: An fMRI study. Iranian Journal of Radiology, 14(2), e17875. [DOI:10.5812/iranjradiol.17875]
Langfitt, J. T., \& Rausch, R. (1996). Word-finding deficits persist after left anterotemporal lobectomy. Archives of Neurology, 53(1), 72-6. [DOI:10.1001/archneur.1996.00550010090021] [PMID]

Loddenkemper, T. (2008). The Wada vanishes-Commentary on Baxendale et al. Epilepsia, 49(4), 724-5. [DOI:10.1111/j.15281167.2008.01515_4.x

Lohmann, H., Deppe, M., Jansen, A., Schwindt, W., \& Knech S. (2004). Task repetition can affect functional magnetic resonance imaging-based measures of language lateralization and lead to pseudoincreases in bilaterality. Journal of Cerebral Blood Flow \& Metabolism, 24(2), 179-87. [DOI:10.1097/01. WCB.0000100066.36077.91]

Loring, D. W. \& Meador, K. J. (2015). The Wada Test: Current Perspectives and Applications. In: Barr W., Morrison C (eds) Handbook on the Neuropsychology of Epilepsy. Clinical Handbooks in Neuropsychology. Springer, New York. [DOI:10.1007/978-0-387-92826-5_5]

Loughman, A., Seneviratne, U., Bowden, S. C., \& D'Souza, W. J. (2016). Epilepsy beyond seizures: Predicting enduring cognitive dysfunction in genetic generalized epilepsies. Epilepsy $\mathcal{E}$ Behavior, 62, 297-303. [DOI:10.1016/j.yebeh.2016.07.010] [PMID]

Mahdavi, A., Saberi, H., Rezvanizadeh, A. R., Lavasani, A. Nilipour, R., \& Oghabian, M. A., et al. (2008). Functional imaging of Broca's area in native Persian speakers: An fMRI study. Iranian Journal of Radiology, 5(4), 215-20. https:/ / www. sid.ir/en/Journal/ViewPaper.aspx?ID=128501

Mahdavi, A., Saberi, H., Houshmand, S., Rezvanizadeh, A., Lavasani, A., \& Nilipour, R., et al. (2010). Cortical representation of Persian word production: An fMRI study. Archives of Iranian Medicine, 13(3), 223-9. https://www.sid.ir/en/Journal/ViewPaper.aspx?ID=171798

Martin, R. C., Loring, D. W., Meador, K. J., Lee, G. P. (1990) The effects of lateralized temporal lobe dysfunction on formal and semantic word fluency. Neuropsychologia, 28, 823-9. [DOI:10.1016/0028-3932(90)90006-A]

Mbwana, J., Berl, M. M., Ritzl, E. K., Rosenberger, L., Mayo, J., \& Weinstein, S., et al. (2009). Limitations to plasticity of language network reorganization in localization related epilepsy. Brain, 132(Pt 2), 347-56. [DOI:10.1093/brain/awn329] [PMID] [PMCID]

Mehvari Habibabadi, J., Saba, N., Barekatain, M., Tabrizi, N. \& Manoochehri, N. (2018). Establishment of a database for drug-resistant epilepsy, Isfahan comprehensive Epilepsy Center, Iran. Journal of Isfahan Medical School, 36(487), 764-70. https://www.researchgate.net/publication/327829681_Establishment_of_a_Database_for_Drug-Resistant_Epilepsy_ Isfahan_Comprehensive_Epilepsy_Center_Iran

Habibabadi, J. M., Moein, H., Basiratnia, R., Badihian, S., Zaki, B., \& Manouchehri, N., et al. (2019). Outcome of lesional epilepsy surgery: Report of the first comprehensive epilepsy program in Iran. Neurology: Clinical Practice, 9(4), 286-95. [DOI:10.1212/ CPJ.0000000000000627] [PMID] [PMCID]

Momenian, M., Nilipour, R., Ghafar Samara, R., Oghabian, M. A., \&Cappa, S. (2016). Neural correlates of verb and noun processing: An fMRI study of Persian. Journal of Neurolinguistics, 37, 12-21. [DOI:10.1016/j.jneuroling.2015.07.003] 
Ni, B., Wang, X., Yu, T., Wu, R., \& Wang, B. (2019). Pre-surgical Language Mapping in Epilepsy: Using fMRI in ChineseSpeaking Patients. Frontiers in Human Neuroscience, 13, 183. [DOI:10.3389/fnhum.2019.00183] [PMID] [PMCID]

Nilipour, R. (2018) (Eds). Neurolinguistics of Persian in Anousha Sedighi and Pouneh Shabani-Jadidi. Oxford: Oxford University press. [DOI:10.1093/oxfordhb/9780198736745.013.18]

Nilipour, R. (2002). Aphasia Picture Naming Battery. Tehran University of Social Welfare \& Rehabilition Sciences Press.

Nilipour, R., Pourshahbaz, A., \& Ghoreyshi, Z. S. (2014). Reliability and validity of bedside version of Persian WAB (PWAB-1). Basic and Clinical Neuroscience, 5(4), 253-8. [PMCID] [PMID]

Nilipour, R., \& Paradis, M. (1995). Breakdown of functional categories in 3 Farsi- English bilingual aphasic patients. In M. Paradis (Eds.) Aspects of Bilingual Aphasia. Oxford: Pergamon Press.

Nilipour, R., \& Ashayeri, H. (1989). Alternating antagonism between two languages with successive recovery of a third in a trilingual aphasic patient. Brain and Language, 36(1), 23-48. [DOI:10.1016/0093-934X(89)90050-3]

Nilipour, R., Bakhtiar, M., Momenian, M., \& Weekes, B. S (2017). Object and action picture naming in brain-damaged Persian speakers with aphasia. Aphasiology, 31(4), 388-405. [D OI:10.1080/02687038.2016.1234583]

Nilipour, R. (2008) (Eds). Aspects of agrammatic language in Persian. In S. Karimi, V. Samiian, \& D. Stilo, Aspects of Iranian Linguistics. London: Cambridge Pub.

Pakdaman, H., Amini Harandi, A., Ashrafi, F. \& Alaeddini, F. (2018). Survey of Epilepsy in Iran. Paper presented at the $15^{\text {th }}$ International Congress on Epilepsy, Tehran, Iran, 23-25 January 2018. https://en.civilica.com/doc/852165/

Paradis, M., Paribakhat, T., \& Nilipour, R. (1987) Bilingual Aphasia Test (Farsi Version). Retrieved from https:/ /www. mcgill.ca/linguistics/research/bat

Peck, K. K., Wierenga, C. E., Moore, A. B., Maher, L. M., Gopinath, K., \& Gaiefsky, M., et al. (2004). Comparison of baseline conditions to investigate syntactic production using functional magnetic resonance imaging. NeuroImage, 23(1), 104-10. https://www.sciencedirect.com/science/article/abs/pii/ S1053811904002733

Petersen, S. E., Fox, P. T., Posner, M. I., Mintun, M., Raichle, M. E. (1988). Positron emission tomographic studies of the cortical anatomy of single-word processing. Nature, 331, 585-9. [DOI:10.1038/331585a0] [PMID]

Peelle, J. E. (2012). The hemispheric lateralization of speech processing depends on what "speech" is: A hierarchical perspective. Frontiers in Human Neuroscience, 6, 309. [DOI:10.3389/ fnhum.2012.00309] [PMID] [PMCID]

Poeppel, D. (2014). The neuroanatomic and neurophysiological infrastructure for speech and language. Current Opinion in Neurobiology, 28, 142-9. [DOI:10.1016/j.conb.2014.07.005] [PMID] [PMCID]

Poeppel, D., \& Hickok, G. (2004). Towards a new functional anatomy of language. Cognition, 92(1-2), 1-12. [DOI:10.1016/j. cognition.2003.11.001] [PMID]
Pujol, J., Deus, J., Losilla, J. M., \& Capdevila, A. (1999). Cerebral lateralization of language in normal left-handed people studied by functional MRI. Neurology, 52(5), 1038. [DOI:10.1212/ WNL.52.5.1038]

Price, C. J. (2012). A review and synthesis of the first 20 years of PET and fMRI studies of heard speech, spoken language and reading. Neuroimage, 62(2), 816-47. [DOI:10.1016/j.neuroimage.2012.04.062] [PMID] [PMCID]

Rauschecker, J. P., \& Scott, S. K. (2009). Maps and streams in the auditory cortex: Nonhuman primates illuminate human speech processing. Nature Neuroscience, 12(6), 718-24. [DOI:10.1038/nn.2331] [PMID] [PMCID]

Risse, G. L. (2006). Cognitive outcomes in patients with frontal lobe epilepsy. Epilepsia, 47, 87-9. [DOI:10.1111/j.15281167.2006.00699.x]

Sabsevitz, D. S., Swanson, S. J., Hammeke, T. A., Spanaki, M. V., Possing, E. T., \& Morris, G. L., et al. (2003). Use of preoperative functional neuroimaging to predict language deficits from epilepsy surgery. Neurology, 60(11), 1788-92. [DOI:10.1212/01. WNL.0000068022.05644.01] [PMID]

Samvelian, P. (2018). Specific features of persian syntax: The Ezâfe Construction, Differential Object Marking and Complex Predictaes. In A. Sedighi, \& P. Shabani-Jadidi (Eds.), The Oxford Handbook of Persian Linguistics. Oxford: Oxford University Press.

Sarkis, R. A., Busch, R. M., Floden, D., Chapin, J. S., Kaiman, K. C., \& Jehi, L., et al. (2013). Predictors of decline in verbal fluency after frontlal lobe epilepsy surgery. Epilepsy and Behavior 27(2), 326-9. [DOI:10.1016/j.yebeh.2013.02.015] [PMID]

Sayehmiri, K., Tavan, H., Sayehmiri, F., Mohammadi, I., \& Carson, K. V. (2014). Prevalence of epilepsy in Iran: A meta-analysis and systematic review. Iranian Journal of Child Neurology, 8(4), 9-17. [PMCID] [PMID]

Shewan, C. M., \& Kertesz, A. (1980). Reliability and validity characteristics of the Western Aphasia Battery (WAB). Journal of Speech and Hearing Disorders, 45(3), 308-24. [DOI:10.1044/ jshd.4503.308] [PMID]

Schwarz, M., \& Pauli, E. (2009). Postoperative speech processing in temporal lobe epilepsy: Functional relationship between object naming, semantics and phonology. Epilepsy $\mathcal{E}$ Behavior, 16(4), 629-33. [DOI:10.1016/j.yebeh.2009.09.016] [PMID]

Scheffer, I. E., Berkovic, S., Capovilla, G., Connolly, M. B., French, J., \& Guilhoto, L., et al. (2017). ILAE classification of the epilepsies: position paper of the ILAE commission for classification and terminology. Epilepsia, 58(4), 51221.[DOI:10.1111/ epi.13709] [PMID] [PMCID]

Seghier, M. L. (2008). Laterality index in functional MRI: methodological issues. Magnetic Resonance Imaging, 26(5), 594-601. [DOI:10.1016/j.mri.2007.10.010] [PMID] [PMCID]

Sherman, E. M., Wiebe, S., Fay-McClymont, T. B., Tellez-Zenteno, J., Metcalfe, A., \& Hernandez-Ronquillo, L., et al. (2011) Neuropsychological outcomes after epilepsy surgery: Systematic review and pooled estimates. Epilepsia, 52(5), 857-69. [DOI:10.1111/j.1528-1167.2011.03022.x] [PMID]

Skidmore, C. T. (2016). Adult focal epilepsies. Epilepsy, 22(1), 94 115. [DOI:10.1212/CON.0000000000000290] [PMID] 
Springer, J. A., Binder, J. R., Hammeke, T. A., Swanson, S. J., Frost, J. A., \& Bellgowan, P. S., et al. (1999). Language dominance in neurologically normal and epilepsy subjects: A functional MRI study. Brain, 122(11), 2033-46. [DOI:10.1093/ brain/122.11.2033] [PMID]

Stowe, L. A., Haverkort, M., \& Zwarts, F. (2005). Rethinking the neurological basis of language. Lingua, 115(7), 997-1042. [DOI:10.1016/j.lingua.2004.01.013]

Stark, C. E., \& Squire, L. R. (2001). When zero is not zero: the problem of ambiguous baseline conditions in fMRI. Proceedings of the National Academy of Sciences, 98(22), 12760-6. [DOI:10.1073/pnas.221462998]

Szaflarski, J. P., Gloss, D., Binder, J. R., Gaillard, W. D., Golby, A. J., \& Holland, S. K., et al. (2017). Practice guideline summary: Use of fMRI in the presurgical evaluation of patients with epilepsy: Report of the Guideline Development, Dissemination, and Implementation Subcommittee of the American Academy of Neurology. Neurology, 88(4), 395-402. [DOI:10.1212/ WNL.0000000000003532] [PMID] [PMCID]

Szaflarski, J. P., Binder, J. R., Possing, E. T., McKiernan, K. A., Ward, B. D., \& Hammeke, T. A. (2002). Language lateralization in left-handed and ambidextrous people: fMRI data. $\mathrm{Neu}$ rology, 59(2), 238-44. [DOI:10.1212/WNL.59.2.238]

Tavakoli, M., Barekatain, M., Doust, H. T. N., Molavi, H., Nouri, R. K., \& Moradi, A., et al. (2011). Cognitive impairments in patients with intractable temporal lobe epilepsy. Journal of research in medical sciences: The Official Journal of Isfahan University of Medical Sciences, 16(11), 1466-72. [PMCID] [PMID]

Thivard, L., Hombrouck, J., Du Montcel, S. T., Delmaire, C., Cohen, L., \& Samson, S., et al. (2005). Productive and perceptive language reorganization in temporal lobe epilepsy. NeuroImage, 24(3), 841-51. [DOI:10.1016/j.neuroimage.2004.10.001] [PMID]

You, X., Zachery, A. N., Fanto, E. J., Norato, G., Germeyan, S. C., \& Emery, E. J., et al. (2019). fMRI prediction of naming change after adult temporal lobe epilepsy surgery: Activation matters. Epilepsia, 60(3), 527-38. [DOI:10.1111/epi.14656] [PMID] [PMCID]

Weber, B., Wellmer, J., Schür, S., Dinkelacker, V., Ruhlmann, J., \& Mormann, F., et al. (2006). Presurgical language fMRI in patients with drug-resistant epilepsy: Effects of task performance. Epilepsia, 47(5), 880-6. [DOI:10.1111/j.15281167.2006.00515.x] [PMID]

WadaJ, R. T. (1960). Intracarotid injection of sodium amytal for lateralization of cerebral speech dominance. Journal of Neurosurgery, 17, 226-32. [DOI:10.3171/jns.1960.17.2.0266]

Wiebe, S. (2000). Epidemiology of temporal lobe epilepsy. Canadian Journal of Neurological Sciences, 27(1), S6-S10. [DOI:10.1017/S0317167100000561] [PMID]

Wiebe, S., Blume, W. T., Girvin, J. P., Eliasziw, M. (2001). A randomized, controlled trial of surgery for temporal-lobe epilepsy. The New England Journal of Medicine, 345(5), 311-8. [DOI:10.1056/NEJM200108023450501] [PMID]

Wilson, S. J., Baxendale, S., Barr, W., Hamed, S., Langfitt, J., \& Samson, S., et al. (2015). Indications and expectations for neuropsychological assessment in routine epilepsy care: Report of the ILAE Neuropsychology Task Force, Diagnostic Methods Commission, 2013-2017. Epilepsia, 56(5), 674-81. [DOI:10.1111/epi.12962] [PMID]
Zhang, J., Liu, W.,Chen, H., Xia, H., Zhou, Z., \& Mei, SH., et al. (2013). Identification of common predictors of surgical outcomes for epilepsy surgery. Neuropsychiatric Disease and Treatment, 9, 1673-82. [DOI:10.2147/NDT.S53802] [PMID] [PMCID] 